\title{
Apoptosis-inducing factor and caspase-dependent apoptotic pathways triggered by different grape seed extracts on human colon cancer cell line Caco-2
}

\author{
Simona Dinicola ${ }^{1,2}$, Alessandra Cucina ${ }^{3}$, Alessia Pasqualato ${ }^{1,2}$, Sara Proietti $^{1}$, Fabrizio D'Anselmi ${ }^{3}$, \\ Gabriella Pasqua ${ }^{4}$, Anna Rita Santamaria ${ }^{4}$, Pierpaolo Coluccia ${ }^{3}$, Aldo Laganà ${ }^{5}$, Donato Antonacci ${ }^{6}$, \\ Alessandro Giuliani ${ }^{7}$ and Mariano Bizzarri ${ }^{1 *}$ \\ ${ }^{1}$ Department of Experimental Medicine, Sapienza University, Viale Regina Elena 324, Roma, Italy \\ ${ }^{2}$ KELL SRL, Via Ennio Quirino Visconti 8, Roma, Italy \\ ${ }^{3}$ Department of Surgery 'Pietro Valdoni', Sapienza University, Via A. Scarpa 14, Roma, Italy \\ ${ }^{4}$ Department of Plant Biology, Sapienza University, Piazzale Aldo Moro 5, Roma, Italy \\ ${ }^{5}$ Department of Chemistry, Sapienza University, Piazzale Aldo Moro 5, Roma, Italy \\ ${ }^{6}$ CRA (Agricultural Research Council), Via Casamassima 148, Turi (Bari), Italy \\ ${ }^{7}$ Department of Environment and Health, Istituto Superiore di Sanità, Viale Regina Elena 299, Roma, Italy
}

(Received 20 October 2009 - Revised 17 March 2010 - Accepted 22 March 2010 - First published online 14 June 2010)

\begin{abstract}
Consumption of grape seed extract (GSE) is widely marketed as a dietary supplement and is considered safe for human health. Nevertheless, the analytical composition of GSE from different grape cultivars, growing in special agronomic constraints, differs greatly in flavan-3-ols content. The major concern with GSE studies is a lack of availability of uniformly standardised preparations, which raises an important question whether different GSE samples have comparable activity and trigger the same mechanisms of action on a given biological system. Therefore, it is tempting to speculate that GSE, obtained from different cultivars, could exert differentiated anticancer effects. The focus of the present study is to determine the selective biological efficacy of GSE obtained from three different sources on the human colon cancer cell line Caco-2. Irrespective of its source, high doses of GSE induced a significant inhibition on Caco-2 cell growth. Moreover, apoptosis was enhanced through both caspase-dependent and caspase-independent mechanisms, leading to an early apoptosis-inducing factor release and, further, to a dramatic increase in caspase 7 and 3 activity. However, a significant difference in apoptotic rates induced by the three grape sources clearly emerged when treating cancer cells with low and intermediate GSE concentrations $(25$ and $50 \mu \mathrm{g} / \mathrm{ml})$.
\end{abstract}

Grape seed extracts: Apoptosis-inducing factor: Apoptosis: Flavan-3-ols

Compelling evidence from epidemiological studies has shown that consumption of a fruit and vegetable-based diet significantly reduces the risk of cancer, especially tumours of the digestive tract ${ }^{(1)}$. Consequently, the focus of cancer research in recent years has been shifting towards the isolation and characterisation of potential chemopreventive agents present in fruits and vegetables ${ }^{(2)}$. In this regard, many phytochemicals, such as bioflavonoids, proanthocyanidins and phytooestrogens, have shown promising chemopreventive and/or anticancer efficacy in various cell cultures and animal models ${ }^{(3)}$. Especially, the composite class of condensed tannins or proanthocyanidins ${ }^{(4)}$ has been demonstrated to exert broad-based and outstanding anticancer effects. Furthermore, according to ancient medical traditions, epidemiological studies have confirmed that consumption of foods with a high content of flavan-3-ols (catechins, condensed tannins, gallate derivatives of cyanidins), flavonoids and anthocyanins significantly reduces the risk for degenerative diseases and several kinds of tumours ${ }^{(5,6)}$. Extracts from grape seeds (GSE) are a prominent, rich source of proanthocyanidins, and several medical reports suggest that consumption of grapes and wine could display beneficial chemopreventive effects on degenerative diseases ${ }^{(7)}$. Namely, in several ongoing studies, GSE have been shown to reduce the incidence of carcinogen-induced mammary tumours in rats and skin cancers in mice and to inhibit the growth of human cancer cells both in vitro and in vivo, after transplantation into animals ${ }^{(8-10)}$. A chemopreventive and anticancer efficacy of GSE was also documented in colon cancer $^{(11,12)}$. GSE treatment inactivates in Caco-2 cells the phosphoinositide 3-kinase/protein kinase B (PI3-kinase/PKB) pathway, leading to a concomitant decrease in Bcl-2 antagonist of cell death (BAD), cAMP response element-binding protein (CREB) and forkhead in rhabdomyosarcoma (FKHR) phosphorylation.

Abbreviations: AIF, apoptosis-inducing factor; GSE, grape seed extract; PARP, poly-ADP-ribose polymerase.

* Corresponding author: Professor Mariano Bizzarri, fax + 39 649766603, email mariano.bizzarri@uniroma1.it 
In turn, GSE treatment induces caspase-dependent activation of apoptosis, through increased caspase 3 activity and enhanced cleavage of poly-ADP-ribose polymerase (PARP) ${ }^{(11)}$. This is of paramount importance, bearing in mind that colorectal cancer is the third most common cause of cancer-related mortality in Western countries ${ }^{(13)}$ and that, despite improvements in the management of colon cancer patients, there is little change in survival rates over the past 50 years $^{(14)}$.

Consumption of foods with high GSE content is widely marketed as a dietary supplement and it is considered safe for human health ${ }^{(15)}$. Nevertheless, the analytical composition of GSE from different sources and namely from different grape cultivars, growing in special agronomic constraints, differs greatly in tannin content, as we have previously shown ${ }^{(16)}$. The major concern with GSE studies is a lack of availability of uniformly standardised preparations. As outlined by Agarwal and co-workers ${ }^{(12)}$, this fact raises an important question whether different GSE samples have comparable activity and trigger the same molecular mechanisms on a given biological system. Therefore, it is tempting to speculate that GSE obtained from different cultivars could exert differentiated anticancer effects, through the involvement of distinct signalling pathways. The focus of the present study is to determine the selective biological efficacy of seed and skin flavan-3-ol constituents, obtained from different grape sources on the human colon cancer cell line Caco-2.

\section{Materials and methods}

\section{Cell culture}

The human colorectal cancer cell line Caco-2 was obtained from the European Collection of Cell Cultures (ECACC). Primary human dermal fibroblasts were isolated from healthy dermis by a collagenase type II digestion.

Cells were seeded into $25 \mathrm{~cm}^{2}$ flasks (Falcon; Becton Dickinson Labware, Franklin Lakes, NJ, USA) in Dulbecco's modified Eagle's medium supplemented with $10 \%$ fetal calf serum and antibiotics (penicillin $100 \mathrm{IU} / \mathrm{ml}$, streptomycin $100 \mu \mathrm{g} / \mathrm{ml}$, gentamycin $200 \mu \mathrm{g} / \mathrm{ml}$ ). The cultures were kept at $37^{\circ} \mathrm{C}$ in an atmosphere of $5 \% \mathrm{CO}_{2}$ in air and the medium was changed every third day. At confluence, the cells were subcultured after removal with $0.05 \%$ trypsin-0.01\% EDTA.

\section{Sample preparation and analysis}

Italia white grape, and Palieri and Red Globe red grape cultivars from an experimental vineyard located in the Puglia region (Italy) were kindly provided by the Agricultural Research Council - Research Unit for grape and winegrowing in the Mediterranean environment (CRA-UTV; Turi, BA, Italy). Fresh grape berry samples were skinned, seeds were separated from the pulp and then the skins and seeds were gently wiped with filter paper to eliminate pulp residues. Homogeneous and dry material from skins and seeds was obtained, extracted with methanol, purified and analysed by electrospray ionisation MS according to a previously published method ${ }^{(17)}$. GSE were re-suspended in $70 \%$ ethanol at a concentration of $30 \mathrm{mg} / \mathrm{ml}$ and stored in the dark at $-20^{\circ} \mathrm{C}$. To obtain a $100 \mu \mathrm{g} / \mathrm{ml}$ concentration (the highest concentration of GSE employed in our experiments), GSE stock solutions were diluted 1:300.

\section{Cell proliferation assay}

Caco-2 cells and human dermal fibroblasts were seeded in twelve-well culture plates (Falcon; Becton Dickinson Labware, Franklin Lakes, NJ, USA) at concentrations ranging between $1 \times 10^{4}$ cells/well and $3 \times 10^{4}$ cells/well in a standard medium. After a zero time $\left(\mathrm{T}_{0}\right)$ cell count, the cells were stimulated with $70 \%$ ethanol (1:300, control), or with Italia, Palieri or Red Globe GSE at 25, 50 or $100 \mu \mathrm{g} / \mathrm{ml}$ and incubated at $37^{\circ} \mathrm{C}$ in an atmosphere of $5 \% \mathrm{CO}_{2}$ in air. The cells were then detached from wells by trypsinisation and cell count was performed by a particle count and size analyser (Beckman Coulter, Inc., Fullerton, CA, USA) after 24, 48, 72 and $96 \mathrm{~h}$. For each data point, two replicate wells were used, and every experiment was performed six times.

\section{Apoptotic cell death assay}

Caco-2 cells were cultured at confluence into $25 \mathrm{~cm}^{2}$ flasks (Falcon; Becton Dickinson Labware, Franklin Lakes, NJ, USA) in a standard medium and stimulated with $70 \%$ ethanol (1:300, control) or with Italia, Palieri or Red Globe GSE at 25,50 or $100 \mu \mathrm{g} / \mathrm{ml}$ and incubated at $37^{\circ} \mathrm{C}$ in an atmosphere of $5 \% \mathrm{CO}_{2}$ in air. After $24 \mathrm{~h}$, the cells were trypsinised, washed twice with PBS and stained with fluorescein isothiocyanate-labelled annexin $\mathrm{V}$ and 7-aminoactinomycine-D (7-AAD) according to the manufacturer's instructions (Instrumental Pro3 Laboratory, Cavenago, MI, Italy). Then, the samples were analysed by flow cytometry (EPICS Coulter XL; Beckman Coulter Inc., Fullerton, CA, USA) for the quantification of apoptotic cells. The fluorescence of 20000 events was measured and an excitation wavelength of $488 \mathrm{~nm}$ was used in combination with standard filters to discriminate between the FL1 and FL3 channels, forward scatter and side scatter.

\section{Immunoblot analysis}

Following treatment with GSE at $50 \mu \mathrm{g} / \mathrm{ml}$, Caco- 2 cells were washed twice with ice-cold PBS and scraped in the following lysis buffer: $50 \mathrm{~mm}$-2-amino-2-hydroxymethyl-propane-1,3diol-HCl, pH 7.4; 150 mM-NaCl; 0.2\% NP-40; $1 \%$ 3-[(3-cholamidopropyl)dimethylammonio]-1-propanesulfonate (CHAPS); 2 mM-EDTA dissolved in tetra-distilled water. A mix of protease inhibitors (Complete-Mini Protease Inhibitor Cocktail Tablets; Roche, Mannheim, Germany) was added just before use. Cellular extracts were then sonicated and centrifuged at $14000 \mathrm{rpm}$ for $10 \mathrm{~min}$. The protein content of supernatant fractions was determined by using the Bradford assay. For immunoblot analyses, cellular extracts were separated on SDS-PAGE gels with a concentration of acrylamide specific for the proteins studied. Proteins were blotted onto nitrocellulose membranes (Bio-Rad Laboratories, Hercules, CA, USA) and probed with the following antibodies: anti-apoptosis-inducing factor (AIF) (anti-AIF, sc-5586; Santa Cruz Biotechnology, Inc., Santa Cruz, CA, USA); anti-cleaved PARP (Sigma, St Louis, MO, USA); anti-cleaved caspase 9 (no. 9501S; Cell Signaling Technology, Inc., Boston, MA, USA); anti-cleaved 
caspase 7 (no. 9491S; Cell Signaling Technology, Inc.); anti-cleaved caspase 3 (no. 9661S; Cell Signaling Technology, Inc.); anti- $\alpha$-tubulin (Sigma, St Louis, MO, USA). Antigens were detected with an enhanced chemiluminescence (ECL) kit from Amersham (Amersham Biosciences, Little Chalfont, Bucks, UK) according to the manufacturer's instructions.

\section{Densitometry}

All Western blot images were acquired and analysed through an Imaging Fluor S densitometer (Bio-Rad Laboratories). The optical density of each condition was normalised against the signal of the internal control $\alpha$-tubulin.

\section{Statistical analysis}

Data were expressed as mean values and standard deviations and statistical analysis was performed using ANOVA, followed by the Bonferroni post hoc test. Pearson correlation coefficients between dose and inhibition potential, on both the entire dataset and inside each cultivar, were computed to assess the statistical relevance of the exerted effect at different scales of definition. Factorial ANOVA was applied on the entire dataset to dissect dose and time effects of treatment. The mixed model of errors was adopted (model III), in order to take into consideration a possible mix of fixed and random effects of the analysed sources of variation. Both the general significance of the entire model from randomness (general effect) and single effect significance were estimated. Differences were considered significant at the level of $P<0.05$. Statistical analysis was performed by using GraphPad Instat software (GraphPad Software, Inc., San Diego, CA, USA).

\section{Results}

Grape skin extracts

Both human dermal fibroblasts and colon cancer cells treated with grape skin extracts did not show any significant change in their proliferative rate (data not shown). In addition, no relevant apoptosis was observed in these conditions (data not shown). In conclusion, grape skin extracts seem to be devoid of significant anticancer effects, even if significant amounts of stilbenes and anthocyanins are present. Some reports have outlined that both stilbenes (resveratrol) ${ }^{(18,19)}$ and anthocyanin conjugates ${ }^{(20,21)}$ exhibit growth-inhibitory properties and pro-apoptotic effects against cancer cells. However, our data did not support these results.

\section{Grape seed extracts}

Growth inhibition. Cell proliferation rates were recorded every $24 \mathrm{~h}$, until $96 \mathrm{~h}$. GSE did not induce any detectable growth-inhibitory effect on cultured human dermal fibroblasts (data not shown). Proliferation rates of Caco-2 cells are otherwise significantly reduced by GSE in a dose-dependent manner (Fig. 1). Growth rates display a similar trend in cell populations treated with GSE obtained from the Italia, Palieri and Red Globe cultivars. Nevertheless, focusing on the lowest
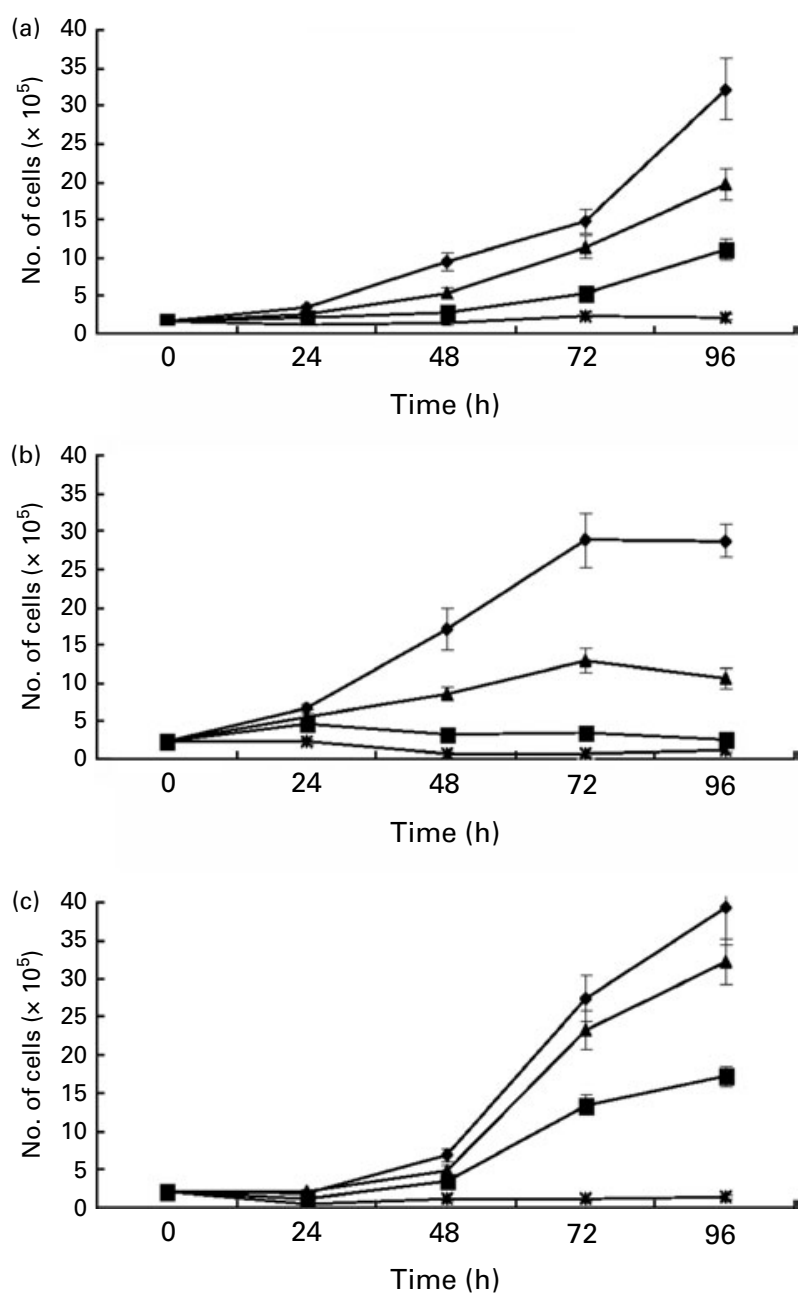

Fig. 1. Effects of grape seed extracts (GSE) compared with control treatment $(--)$ on proliferation of Caco-2 cells after 24, 48, 72 and $96 \mathrm{~h}$. The cells were stimulated with Italia (a), Palieri (b) or Red Globe (c) GSE at $25(-\boldsymbol{\Lambda}-)$, $50(-\square-)$ or $100(*) \mu \mathrm{g} / \mathrm{ml}$. Values are means of six independent experiments performed in duplicate, with standard deviations represented by vertical bars. Data are shown in the Supplementary Tables, available online.

active concentration $(25 \mu \mathrm{g} / \mathrm{ml})$, it clearly emerges that the most active inhibition is attained by GSE from the Italia and Palieri cultivars, after only $24 \mathrm{~h}$. In Italia GSE-treated cells, the growth inhibition is statistically significant at each GSE concentration and at all the times considered $(P<0.01)$, reaching the most relevant inhibition after $96 \mathrm{~h}$ at $100 \mu \mathrm{g} / \mathrm{ml}(93 \% ; P<0.001)$. In Palieri GSE-treated samples, the growth inhibition is statistically significant at every GSE concentration and at all the times studied $(P<0 \cdot 001)$, reaching high inhibitory effects at 50 and $100 \mu \mathrm{g} / \mathrm{ml}$ (91 and $96 \%$, respectively; $P<0.001)$. In Red Globe GSE-treated cells, the growth inhibition is statistically significant at all the times studied only at 50 and $100 \mu \mathrm{g} / \mathrm{ml}(P<0.001)$, reaching the most relevant inhibition at $100 \mu \mathrm{g} / \mathrm{ml}(96 \% ; P<0.001)$. At $25 \mu \mathrm{g} / \mathrm{ml}$, the growth inhibition reaches significant values only at $48 \mathrm{~h}(P<0.01)$ and $96 \mathrm{~h}(P<0.05)$. At the highest GSE concentration $(100 \mu \mathrm{g} / \mathrm{ml})$, differences between the three cultivars disappear and Caco-2 growth is almost completely inhibited after the first $48 \mathrm{~h}$. 
In order to obtain a global comparison among the studied cultivars as for growth inhibition along both exposure time and dose dependency, an ANOVA approach was undertaken.

First, on the entire dataset made by thirty-six independent samples (three doses $\times$ four times $\times$ three cultivars), a statistically significant Pearson correlation $(r 0.782 ; P<0.0001)$ with dose was observed, pointing to a global effect of all the cultivars.

The presence of a statistically significant dose-inhibition relationship is observed for each single cultivar (Italia, $r$ 0.868, P<0.0003; Palieri, $r$ 0.625, P<0.03; Red Globe, $r 0.959 ; P<0.001)$.

It is worth noting that the Pearson correlation simply measures the goodness of fit of the scattering of the points in the dose-inhibition plane to a straight line without being explicitly linked to the slope of the line, which is analysed by a general linear model (ANOVA) where effects of time are inserted as well. The whole set gives the results showed in Table 1 as for the significance of both time and dose effects.

Both time and dose enter with a statistically significant load into the model, so demonstrating the dependency of growth inhibition on both time of exposure and dose. A similar pattern was recorded for each cultivar (data not shown).

When the cultivars were compared with each other by an ANOVA model, we show that they are different as for general inhibition effect while they do not reach statistical significance as for the slope of their dose-inhibition relationship that is present in all the cultivars (Table 2).

The Palieri cultivar was the most effective (average inhibition over all the conditions 70.85), while the Italia and Red Globe cultivars were markedly lower (57.80 and 51.25, respectively).

The order of effect scales with the amount of procyanidins, with the highest procyanidin content paralleled by the most marked effect. In our experimental setting we cannot statistically separate the effect of procyanidins from the general effect of cultivar; nevertheless we can hypothesise a possible involvement of this class of substances in growth inhibition.

Apoptosis. The possible apoptotic effect of GSE on the colorectal cancer cells was next examined by annexin $\mathrm{V}$ and 7-aminoactinomycine-D (7-AAD) staining, where cells were treated with GSE $(25,50,100 \mu \mathrm{g} / \mathrm{ml})$ for $24 \mathrm{~h}$ under similar conditions as in cell growth studies. Above the threshold value of $50 \mu \mathrm{g} / \mathrm{ml}$, GSE treatment showed a roughly significant dose-dependent increase in apoptotic cell population (Fig. 2(a) and (b)). Normal human fibroblasts cultured with GSE did not show any significant modification in programmed cell death levels (data not shown). In Italia, Palieri and Red Globe GSE-treated cells apoptosis is higher in respect to both control and camptothecin-treated control samples at 50

Table 1. ANOVA of time- and dose-related growth inhibition

\begin{tabular}{lccc}
\hline & $F$ & $P$ & $R^{2}$ \\
\hline $\begin{array}{l}\text { General effects } \\
\text { Dependent variable: growth inhibition }\end{array}$ & 43.84 & $<0.0001$ & 0.7266 \\
Single effects & & & \\
$\quad$ Source & & & \\
$\quad$ Time & 3.75 & 0.0007 & \\
$\quad$ Dose & 8.58 & 0.0001 & \\
\hline
\end{tabular}

Table 2. ANOVA of cultivar and dose-related growth inhibition

\begin{tabular}{lrrr}
\hline & $F$ & $P$ & $R^{2}$ \\
\hline $\begin{array}{l}\text { General effects } \\
\text { Dependent variable: growth inhibition }\end{array}$ & 17.44 & $<0.0001$ & 0.663 \\
$\begin{array}{l}\text { Single effects } \\
\quad \text { Source } \\
\quad \text { Cultivar }\end{array}$ & & & \\
$\quad$ Dose & 5.92 & 0.0068 & \\
\hline
\end{tabular}

and $100 \mu \mathrm{g} / \mathrm{ml} \quad(P<0 \cdot 001)$. At $25 \mu \mathrm{g} / \mathrm{ml}$, only Palieri GSE-treated cells showed a statistically significant increase in apoptotic rate with respect to control. At the highest GSE concentration $(100 \mu \mathrm{g} / \mathrm{ml})$, in all experimental samples the highest apoptotic values were reached, but without statistically significant differences between the three cultivars. At the highest concentrations of GSE, an increase in late apoptosis was observed (Fig. 2(b)). In both Italia- and Red Globe-treated cells, the rate of late apoptosis was noteworthy at a concentration of $100 \mu \mathrm{g} / \mathrm{ml}$, while in Palieri-treated samples, it became evidently high at $50 \mu \mathrm{g} / \mathrm{ml}$.

Molecular parameters. Molecular parameters were evaluated only on samples treated with GSE at $50 \mu \mathrm{g} / \mathrm{ml}$. This choice was suggested by the fact that Caco- 2 cells treated with GSE at $100 \mu \mathrm{g} / \mathrm{ml}$ gave paradoxical results, i.e. reduced values of the overall apoptotic markers, despite an increased apoptotic rate, were observed in these conditions. As previously observed in DU145 human prostate carcinoma cells treated with GSE fractions ${ }^{(22)}$, one possible explanation could be that the $100 \mu \mathrm{g} / \mathrm{ml}$ dose of GSE produces massive apoptotic cell death (already after $24 \mathrm{~h}$ ), making the analyses of caspases and PARP cleavages impractical in total cell lysates.

Caspases. Caspase 8 activity was not significantly modified in GSE-treated cells (data not shown). On the contrary, an increase in caspase 9 was observed already from the first hours in GSE-treated Caco-2 cells and it reached the maximum at $24 \mathrm{~h}$ (Fig. 3(a)). Nevertheless, terminal effector caspases (caspases 3 and 7) did not increase until $24 \mathrm{~h}$. It must be emphasised that both caspases 7 and 3 increase up to 5-fold in comparison with the basal values (Fig. 3(b) and (c)). This trend behaved apparently similar for all tested cultivars, even if absolute values differed greatly for each grape type. The highest increase in caspases activity was observedas expected - in Caco- 2 cells treated with GSE obtained from the Palieri cultivar. These data suggest that GSE trigger apoptosis through the intrinsic caspase-apoptotic pathways ${ }^{(23)}$.

Apoptosis-inducing factor. In all tested samples, AIF showed the same trend: it increased earlier (at $6 \mathrm{~h}$ ) than caspases and it reached the highest value at $24 \mathrm{~h}$ (Fig. 4(a)). Absolute values were significantly higher in samples treated with GSE obtained from the Italia cultivar.

Cleaved poly-ADP-ribose polymerase. A similar trend was observed for cleaved PARP values: an increase of cleaved PARP was observed at $3 \mathrm{~h}$ after GSE treatment. The highest levels were reached at $24 \mathrm{~h}$ (Fig. 4(b)). As for caspases 3 and 7, the highest absolute concentration was observed in Caco- 2 cells treated with Palieri-derived GSE. The earliest increase in cleaved PARP seems to suggest that early apoptosis was triggered by AIF - thought to be a caspase-independent apoptotic pathway - and further 
(a)

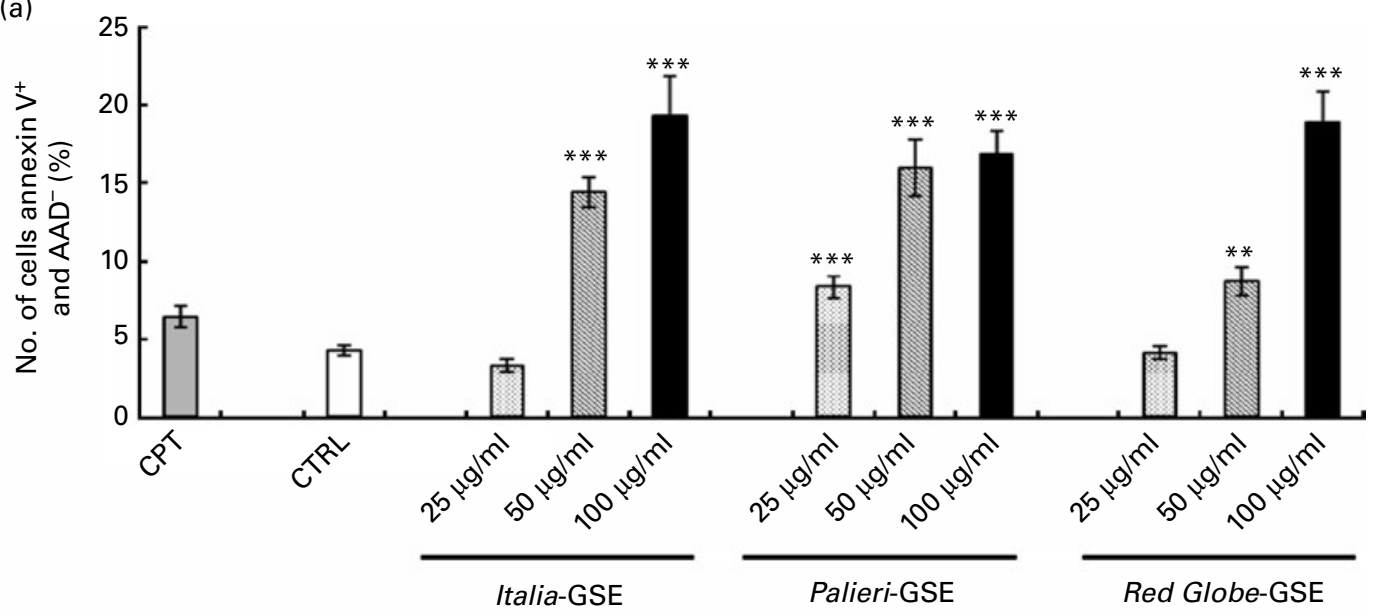

(b)
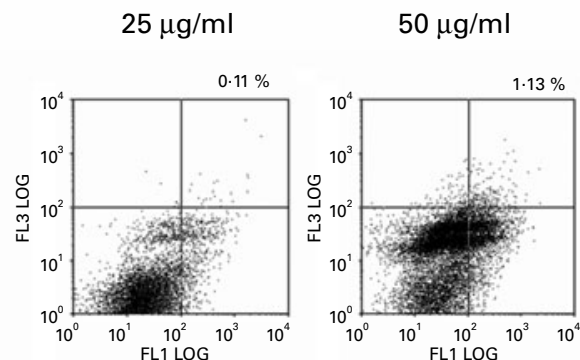

$100 \mu \mathrm{g} / \mathrm{ml}$
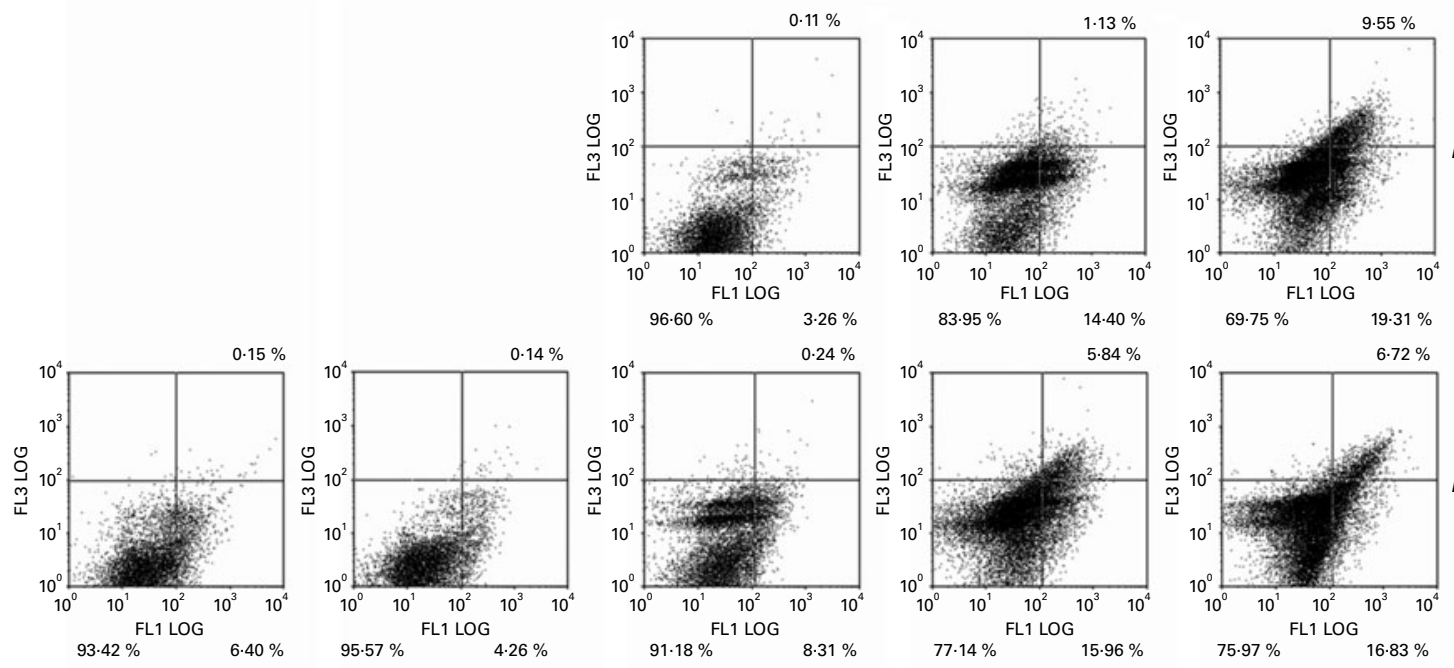

Italia-GSE
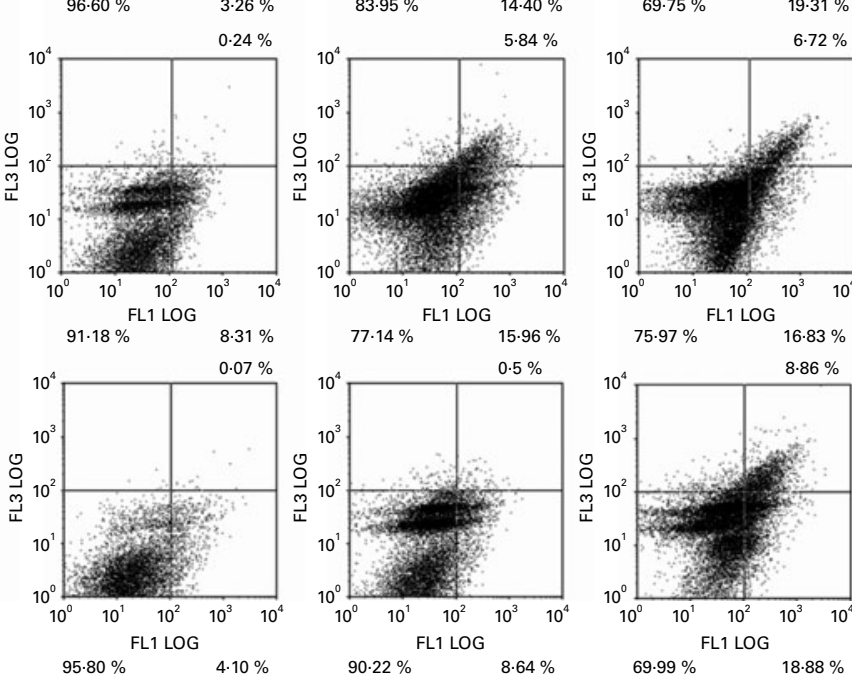

Palieri-GSE

Red Globe-GSE

Fig. 2. (a) Effects of grape seed extracts (GSE) on apoptosis of Caco-2 cells after $24 \mathrm{~h}$. Values are means of three independent experiments, with standard deviations represented by vertical bars. Mean value was significantly different from that for the control (CTRL) treatment: ${ }^{* *} P<0.01,{ }^{* * *} P<0.001$. 7-AAD, 7-aminoactinomycine-D; CPT, camptothecin. (b) Dual-parameter flow cytometric density dot plots for GSE-treated Caco-2 cells. Fluorescence intensity for annexin $\mathrm{V}$-fluorescein isothiocyanate is plotted on the $x$-axis and 7-AAD is plotted on the $y$-axis. The lower left quadrant cells (annexin $V^{-} / 7-A A D^{-}$) were defined as viable cells, the lower right quadrant cells (annexin $\mathrm{V}^{+} / 7-\mathrm{AAD}^{-}$) as apoptotic cells, and the upper right quadrant cells (annexin $\mathrm{V}^{+} / 7-\mathrm{AAD}^{+}$) as late apoptotic cells

enhanced by increased activation of the terminal caspase effectors, i.e. caspases 3 and 7.

\section{Discussion}

In the last 10 years, several studies have convincingly documented the anticancer and cancer-chemopreventive efficacy of GSE against various cancers ${ }^{(24-26)}$; however, only few studies have investigated the anticancer effects exerted by
GSE on human colon tumours ${ }^{(11,15,27,28)}$. Moreover, a major caveat has been the composition of various GSE preparations being marketed under different names, and those being used under laboratory conditions. The lack of standardised preparations has limited the validity and translational potential of the research findings obtained in the laboratory setting using different preparations or sources of GSE. As previously shown by our laboratory, GSE obtained from different cultivars, and even GSE provided by the same cultivars, grown 
Italia-GSE

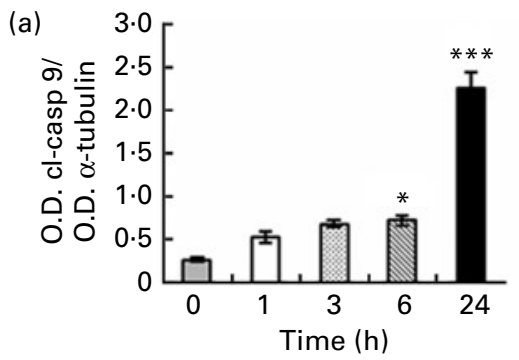

cl-casp 9

$\alpha$-Tubulin

(b)

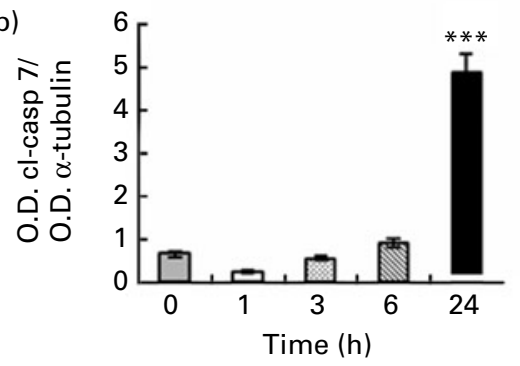

cl-casp 7

$\alpha$-Tubulin

(c)

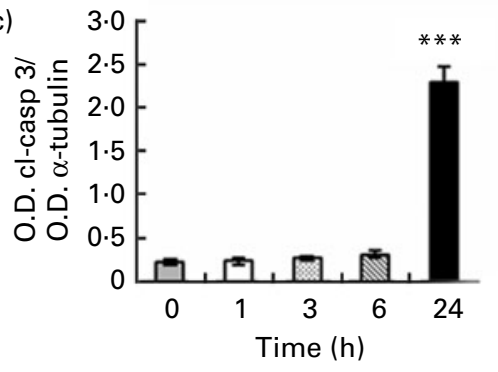

cl-casp 3

$\alpha$-Tubulin
Palieri-GSE
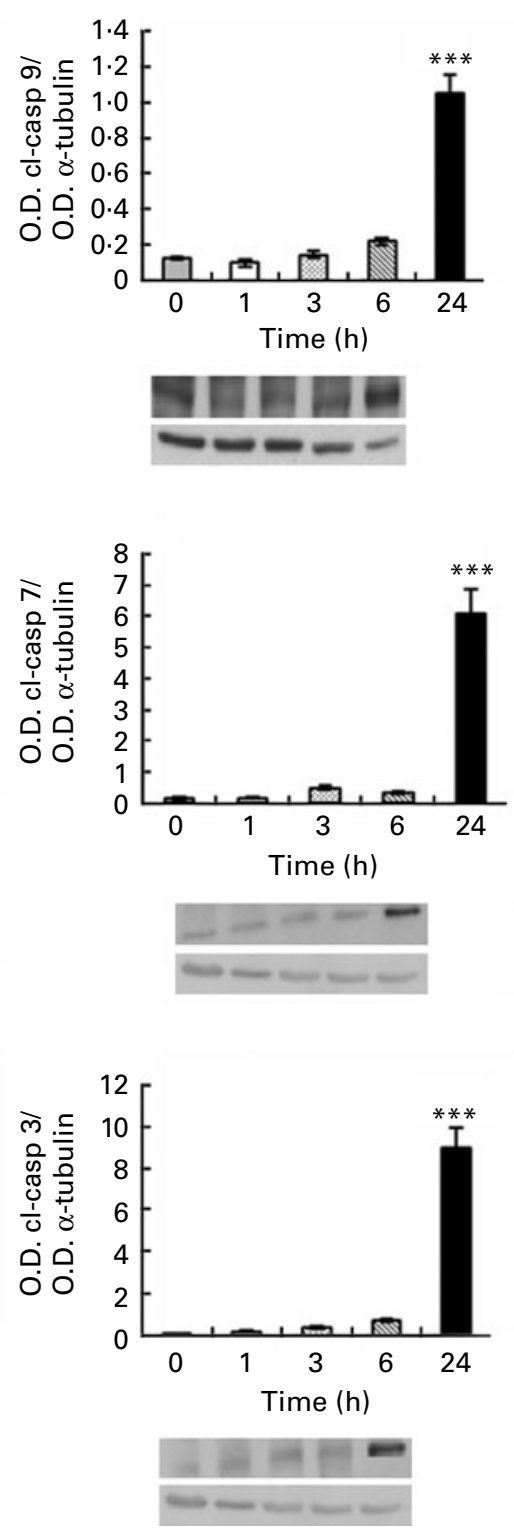

Red Globe-GSE
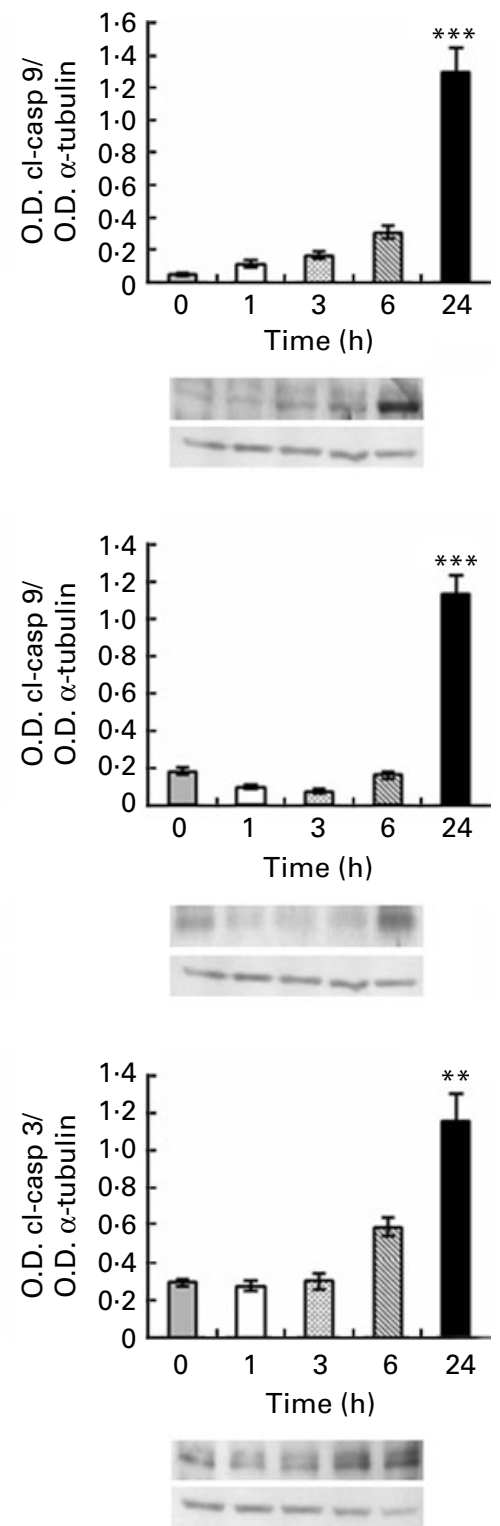

Fig. 3. Immunoblots showing the expression of cleaved caspase (cl-casp) 9 (a), cl-casp 7 (b) and cl-casp 3 (c) in Caco-2 cells treated with Italia, Palieri and Red Globe grape seed extracts (GSE) from 0 to $24 \mathrm{~h}$. Data represent densitometric quantification of optical density (O.D.) of specific protein signal normalised with the O.D. values of $\alpha$-tubulin, served as a loading control. Values are means $(n 3)$, with standard deviations represented by vertical bars. Mean value was significantly different from that for the control treatment: ${ }^{*} P<0.05$, ${ }^{\star *} P<0.01$, ${ }^{\star \star \star} ~ P<0.001$.

under different agronomical conditions, display a remarkable diversity in their composition ${ }^{(16)}$. To address some of these issues, we compared the biological effects of GSE procured from three different cultivars (Italia, Palieri and Red Globe) against the human colon cancer cell line Caco- 2 .

Irrespective of its source, GSE produce strong biological effects on Caco-2 cells, which include growth inhibition, induction of markers of apoptotic signalling pathways, and programmed cell death. Growth inhibition becomes evident already from the first $24 \mathrm{~h}$ of treatment and progressively increases. This trend is quite similar in all experiments and seems to be independent from the type of GSE studied, even if the highest inhibitory effect was recorded in the Palieri GSE-treated cells. Growth inhibition is clearly dose-dependent, increasing linearly with the concentration of the grape extract. Independently from the type of cultivar, in samples treated with GSE at $100 \mu \mathrm{g} / \mathrm{ml}$, cell growth is almost completely abolished after the first $24 \mathrm{~h}$.

Loss of apoptotic function is a major contributor towards the resistance of cancer cells to both metabolic (hypoxic, energetic) stresses and cytotoxic treatments. Therefore, it should be highly desirable to diversify the availability of apoptotic and pro-apoptotic substances that could be used in cancer therapy. As previously outlined, GSE not only exert significant inhibitory effects on cancer growth, but also induce a significant programmed cell death response in colon cancer cells. This effect seems to be clearly dose-dependent, with the highest apoptotic rates observed in Caco- 2 cells treated with GSE 

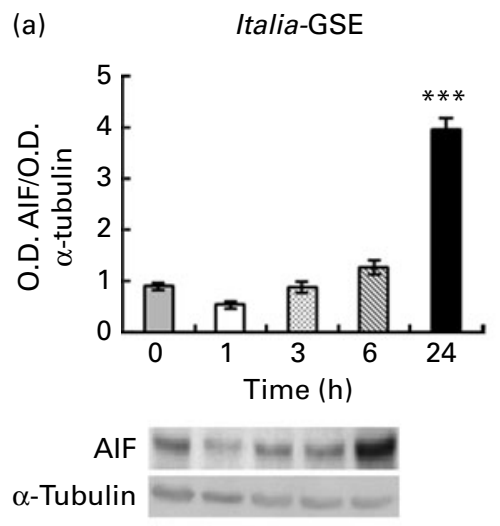

(b)
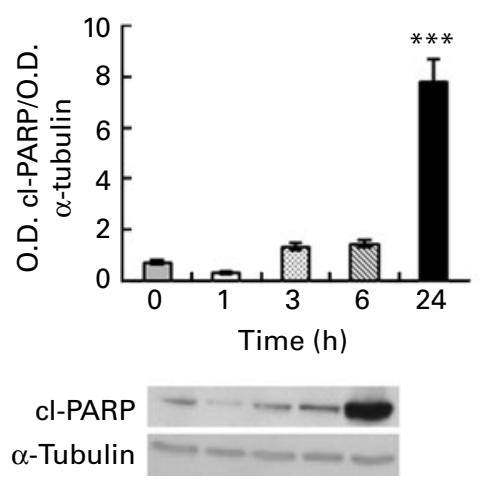

Palieri-GSE
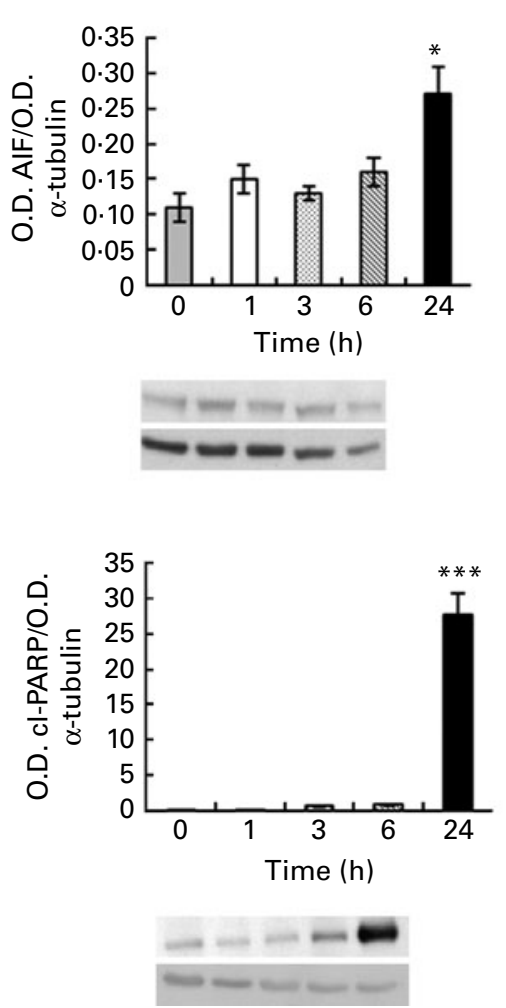

Red Globe-GSE
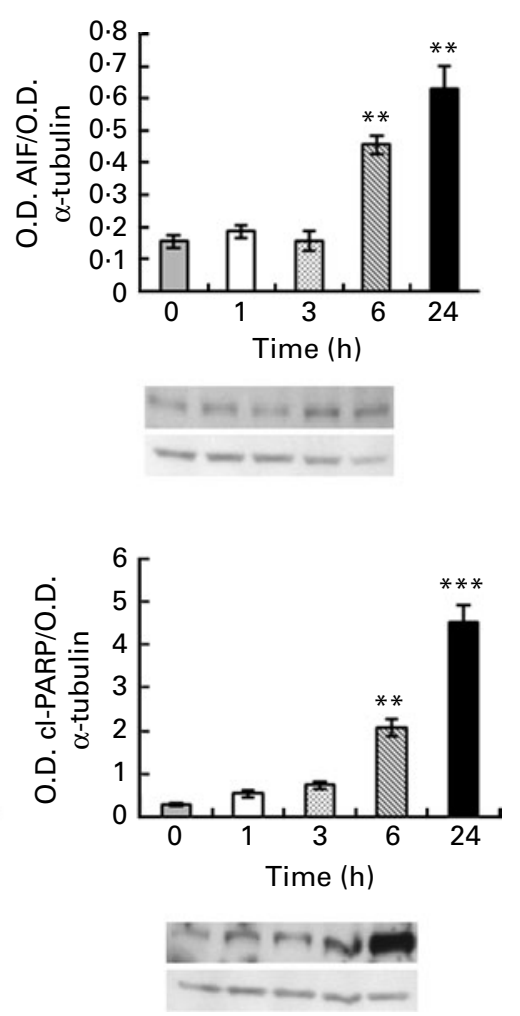

Fig. 4. Immunoblots showing the expression of apoptosis-inducing factor (AIF) (a) and cleaved poly-ADP-ribose polymerase (cl-PARP) (b) in Caco-2 cells treated with Italia, Palieri and Red Globe grape seed extracts (GSE) from 0 to $24 \mathrm{~h}$. Data represent densitometric quantification of optical density (O.D.) of specific protein signal normalised with the O.D. values of $\alpha$-tubulin, served as a loading control. Values are means $(n 3)$, with standard deviations represented by vertical bars. Mean value was significantly different from that for the control treatment: ${ }^{\star} P<0.05,{ }^{\star *} P<0.01,{ }^{\star \star \star} P<0.001$.

at a concentration of $100 \mu \mathrm{g} / \mathrm{ml}$. However, some subtle differences are emerging from the whole picture. In fact, when treating cancer cells with low and intermediate concentrations of GSE $(25$ and $50 \mu \mathrm{g} / \mathrm{ml})$, Palieri-treated samples showed a significantly higher response compared with the other two cultivars. As expected, both cleaved PARP and caspase 3 activity were significantly enhanced in samples treated with Palieri-derived GSE than in both Red Globeand Italia-treated cells. It is likely that such differences could be explained by a different chemical composition of GSE obtained from the Palieri cultivar.

Indeed, GSE obtained from our different sources showed significant diversities in flavan-3-ol composition, analysed by means of the liquid chromatography-MS technique, as previously reported ${ }^{(17)}$.

Both stilbenes and anthocyanin conjugates are represented at high concentrations in skin extracts (with the exception of the white grape cultivar, Italia); meanwhile, they are recorded only in traces in extracts obtained from seeds. No appreciable quantities of gallic acid have been found both in skin and seed extracts. On the contrary, flavan-3-ols - procyanidin B1 and B2, procyanidin dimers, catechin, epicatechin and epigallocatechin-gallate - are highly represented only in $\mathrm{GSE}^{(17)}$. However, GSE obtained from the three cultivars greatly differ in their pyrogallol-type structure-containing compounds: catechin and epicatechin gallate, procyanidin dimers, trimers and tetramers, procyanidins gallate, collectively known as proanthocyanidins. Proanthocyanidins are naturally occurring polyphenolic flavan-3-ols with different chemical structure, pharmacology and characteristics, widely distributed in plants ${ }^{(29)}$. Polymeric and oligomeric proanthocyanidins (also called condensed tannins) are polyphenols composed of chains of flavan-3-ol units, (+)-catechin and (-)-epicatechin linked through $\mathrm{C} 4-\mathrm{C} 6$ and $\mathrm{C} 4-\mathrm{C} 8$ interflavan bonds. Oligomeric proanthocyanidins are the only macromolecular constituents present in GSE, which contain variable amounts of monomeric catechin and epicatechin chains ${ }^{(14,30)}$.

It has been suggested that flavan-3-ols can exert anticancer activity when they are provided by a pyrogallol-type structure $^{(31)}$. In fact, previous reports have evidenced that epigallocatechin gallate induces apoptosis in colon cancer ${ }^{(32)}$, while this induction was very weak by catechin and epicatechin, which lack a galloyl group ${ }^{(33,34)}$, suggesting a certain structure-function relationship in apoptosis-inducing activity. Therefore, it is likely that a pyrogallol-type structure in a B-ring may contribute to the apoptosis-inducing activity. Indeed, the highest concentration of compounds provided by a pyrogallol-type structure was observed in Palieri GSE ${ }^{(17)}$ and, as expected, the most significant apoptotic rate was obtained in Palieri GSE-treated samples.

It must be emphasised that these results are strictly dependent on the cancer cell line studied and they should not to be extrapolated to other types of colon cancers.

The apoptotic rate induced by GSE on Caco- 2 cells is lower than that recorded in other tumours (prostate, leukaemia), but is comparable with the results obtained by Agarwal and 
co-workers on several colon cancer cell lines (SW480, HT29 and LoVo) ${ }^{(12)}$ and by Kim et al. on SNU-C4 tumour cells ${ }^{(35)}$. Even if GSE-induced apoptosis is likely to be p53-independent (though Caco-2 cells are p53 defective), apoptosis is triggered through a caspase-dependent mechanism, leading to the activation of two caspase effectors, caspases 3 and 7. This result confirms previous data ${ }^{(11)}$, indicating that apoptosis induced by GSE belongs to the intrinsic apoptotic pathway, though caspase 8 levels in GSE-treated cancer cells are unmodified. The caspase-dependent pathway might not be the only apoptotic mechanism triggered by GSE, bearing in mind that a rise in cleaved PARP - even if it is not always statistically significant - can be recorded before an increase in caspase activity is observed. Indeed, AIF, known to induce apoptosis via a caspase-independent mechanism, increases early in GSE-treated samples. Even if two previous studies ${ }^{(36,37)}$ demonstrated the involvement of AIF-mediated apoptosis in cancer cells treated with epigallocatechin gallate, until now no data have been published about the possibility that GSE could trigger a similar caspase-independent apoptotic pathway in colon cancer. For the first time we demonstrated that GSE enhance a significant AIF release in colon cancer cells together with an increase in caspase activity. These results suggest that GSE-induced apoptosis in Caco-2 cells can be considered a biphasic process, obtained through both caspase-dependent and caspase-independent pathways.

\section{Acknowledgements}

The present study was supported by the Italian Ministry of Agriculture (MIPAF-VANSUT program; grant no. 8.1.2.2.7.8).

S. D. designed and performed the experiments, analysed the data and wrote the manuscript. A. C. contributed to the design of the experiments and gave conceptual advice. A. P., S. P. and F. D'A. contributed to the performance of the experiments and analysis of the data. G. P., A. R. S. and A. L. prepared and chemically characterised the GSE. P. C. performed cytofluorimetric analysis. D. A. provided grape seeds. A. G. statistically analysed output data. M. B. supervised the experiments and wrote the manuscript.

We are grateful to Mrs Luisa Di Renzo for her helpful assistance and for reviewing the manuscript.

All authors have no personal or financial conflicts of interest and they have not entered into any agreement that could interfere with our access to the data on the research or on our ability to analyse the data independently, to prepare manuscripts and to publish them.

The Supplementary Tables are available online only at http://journals.cambridge.org/action/displayJournal?jid=bjn

\section{References}

1. La Vecchia C (2004) Mediterranean diet and cancer. Public Health Nutr 7, 965-968.

2. Cooke D, Steward WP, Gescher AJ, et al. (2005) Anthocyans from fruits and vegetables - does bright colour signal cancer chemopreventive activity? Eur J Cancer 41, 1931-1940.

3. Surh YJ (2003) Cancer chemoprevention with dietary phytochemicals. Nat Rev Cancer 3, 768-780.
4. Hemingway RW \& Karchesy JJ (1989) Structural variations in proanthocyanidins and their derivatives. In Chemistry and Significance of Condensed Tannins, pp. 47-60 [RW Hemingway and JJ Karchesy, editors]. New York: Plenum Press.

5. Gerhauser C (2008) Cancer chemopreventive potential of apples, apple juice and apple components. Planta Med 74, 1608-1624.

6. Can-Lan S, Jian-Min Y, Woon-Puay K, et al. (2006) Green tea, black tea and breast cancer risk: a meta-analysis of epidemiological studies. Carcinogenesis 27, 1310-1315.

7. Carlson S, Peng N, Prasain JK, et al. (2008) Effects of botanical dietary supplements on cardiovascular, cognitive, and metabolic function in males and females. Gend Med 5A, S76-S90

8. Ye X, Krohn RL, Liu W, et al. (1999) The cytotoxic effects of a novel IH636 grape seed proanthocyanidin extract on cultured human cancer cells. Mol Cell Biochem 196, 99-108.

9. Singh RP, Tyagi AK, Dhanalakshmi S, et al. (2004) Grape seed extract inhibits advanced human prostate tumor growth and angiogenesis and upregulates insulin-like growth factor binding protein-3. Int J Cancer 108, 733-740.

10. Singletary KW \& Meline B (2001) Effect of grape seed proanthocyanidins on colon aberrant crypts and breast tumors in a rat dual-organ tumor model. Nutr Cancer 39, 252-258.

11. Engelbrecht AM, Mattheyse M, Ellis B, et al. (2007) Proanthocyanidin from grape seeds inactivates the PI3-kinase/PKB pathway and induces apoptosis in a colon cancer cell line. Cancer Lett 258, 144-153.

12. Kaur M, Mandair R, Agarwal R, et al. (2008) Grape seed extract induces cell cycle arrest and apoptosis in human colon carcinoma cells. Nutr Cancer 60, Suppl. 1, 2-11.

13. Landis SH, Murray T, Bolden S, et al. (1999) Cancer statistics. CA Cancer J Clin 49, 8-31.

14. Yeatman TJ \& Chambers AF (2003) Osteopontin and colon cancer progression. Clin Exp Metastasis 20, 85-90.

15. Clifton PM (2004) Effect of grape seed extract and quercetin on cardiovascular and endothelial parameters in high risk subjects. J Biomed Biotechnol 5, 272-278.

16. Cavaliere C, Foglia P, Marini F, et al. (2010) The interactive effects of irrigation, nitrogen fertilization rate, delayed harvest and storage on polyphenol content in grape (Vitis vinifera) berry: a factorial experimental design. J Agric Food Chem (In the Press).

17. Cavaliere C, Foglia P, Gubbiotti R, et al. (2008) Rapidresolution liquid chromatography/mass spectrometry for determination and quantitation of polyphenols in grape berries. Rapid Commun Mass Spectrom 22, 3089-3099.

18. Marel AK, Lizard G, Izard JC, et al. (2008) Inhibitory effects of trans-resveratrol analogs molecules on the proliferation and the cell cycle progression of human colon tumoral cells. Mol Nutr Food Res 52, 538-548.

19. Benitez DA, Pozo-Guisado E, Alvarez-Barrientos A, et al. (2007) Mechanisms involved in resveratrol-induced apoptosis and cell cycle arrest in prostate cancer-derived cell lines. J Androl 28, 282-293.

20. Li L, Adams LS, Chen S, et al. (2009) Eugenia jambolana Lam. berry extract inhibits growth and induces apoptosis of human breast cancer but not non-tumorigenic breast cells. J Agric Food Chem 57, 826-831.

21. Thomasset S, Teller N, Cai H, et al. (2009) Do anthocyanins and anthocyanidins, cancer chemopreventive pigments in the diet, merit development as potential drugs? Cancer Chemother Pharmacol 64, 201-211.

22. Veluri R, Singh RP, Liu Z, et al. (2006) Fractionation of grape seed extract and identification of gallic acid as one of the major active constituents causing growth inhibition and apoptotic death of DU145 human prostate carcinoma cells. Carcinogenesis 27, 1445-1453. 
23. MacKenzie SH \& Clark AC (2008) Targeting cell death in tumors by activating caspases. Curr Cancer Drug Targets 8 , 98-109.

24. Sharma G, Tyagi AK, Singh RP, et al. (2004) Synergistic anticancer effects of grape seed extract and conventional cytotoxic agent doxorubicin against human breast carcinoma cells. Breast Cancer Res Treat 85, 1-12.

25. Nandakumar V, Singh T \& Katiyar SK (2008) Multi-targeted prevention and therapy of cancer by protoanthocyanidins. Cancer Lett 269, 378-387.

26. Joshi SS, Kuszynki CA \& Bagchi D (2001) The cellular and molecular basis of health benefits of grape seed proanthocyanidins extract. Curr Pharmacol Biotechnol 2, 2187-2200.

27. Kaur M, Mandair R, Agarwal R, et al. (2008) Grape seed extract induces cell cycle arrest and apoptosis in human colon carcinoma cells. Nutr Cancer 60, 2-11.

28. Leifert WR \& Abeywardena MY (2008) Grape seed and red wine polyphenol extracts inhibit cellular cholesterol uptake, cell proliferation, and 5-lipoxygenase activity. Nutr Res 28, 842-850.

29. Gabetta B, Fuzzati N, Griffino A, et al. (2000) Characterization of proanthocyanidins from grape seeds. Fitoterapia 71, 162-175.

30. Weber HA, Hodges AE, Guthrie JR, et al. (2007) Comparison of proanthocyanidins in commercial antioxidants: grape seed and pine bark extracts. J Agric Food Chem 55, 148-156.
31. Saeki K, Hayakawa S, Isemura M, et al. (2000) Importance of a pyrogallol-type structure in catechin compounds for apoptosisinducing activity. Phytochemistry 53, 391-394.

32. Inaba H, Nagaoka Y, Kushima Y, et al. (2008) Comparative examination of anti-proliferative activities of (-)-epigallocatechin gallate and (-)-epigallocatechin against HCT116 colorectal carcinoma cells. Biol Pharm Bull 31, 79-84.

33. Achiwa Y, Hibasami H, Katsuzaki H, et al. (1997) Inhibitory effects of persimmon (Diospyros kaki) extract and related polyphenol compounds on growth of human lymphoid leukemia cells. Biosci Biotechnol Biochem 61, 1099-1101.

34. Hibasami H, Komiya T, Achiwa Y, et al. (1998) Induction of apoptosis in human stomach cancer cells by green tea catechins. Oncol Rep 5, 527-529.

35. Kim YJ, Park HJ, Yoon SH, et al. (2005) Anticancer effects of oligomeric proanthocyanidins on human colorectal cancer cell line, SNU-C4. World J Gastroenterol 11, 4674-4678.

36. Nakazato T, Ito K, Ikeda Y, et al. (2000) Green tea component, catechin, induces apoptosis of human malignant B cells via production of reactive oxygen species. Cancer Res 11, 6040-6049.

37. Wu PP, Kuo SC, Huang WW, et al. (2009) (-)-Epigallocatechin gallate induced apoptosis in human adrenal cancer NCIH295 cells through caspase-dependent and caspase-independent pathway. Anticancer Res 29, 1435-1442. 